\title{
Orale Kontrazeption und Krebs
}

Hunderte Millionen von Frauen verhüten weltweit mittels oraler Kontrazeption. Ein möglicher Einfluss auf das Krebsrisiko wurde von Beginn an diskutiert, galt bislang aber als nicht ausreichend untersucht. Zwei neue große Studien sollten die Sachlage nun endgültig klären.

Nach Schätzungen nehmen 100 bis 150 Millionen Frauen weltweit orale Kontrazeptiva ein. Seit Beginn der Einführung dieser Verhütungsmittel (in den 1960er-Jahren) wurde ein möglicher Zusammenhang zwischen der langjährigen Einnahme und einem erhöhten oder erniedrigten Krebsrisiko untersucht. Laut verfügbarer Evidenz haben Anwenderinnen von kombinierten oralen Kontrazeptiva ein erhöhtes Risiko für Brustkrebs und Zervixkarzinom, während gleichzeitig das Risiko für Endometrium- und Ovarialkarzinom geringer sein soll; Frauen, die aktuell die Pille nehmen, sollen außerdem vor Kolorektalkarzinom geschützt sein, berichten Forscher der Universität Aberdeen in Schottland [1]. Zwei Fragen seien allerdings noch nicht ausreichend geklärt:

1. Wie lange hält der Nutzen bezüglich Endometrium-, Ovarial- und Kolorektalkarzinom an?

2. Sind mit der langjährigen Einnahme auch Risiken für andere Krebsformen in späteren Lebensabschnitten verbunden?

Nutzen des niedrigeren Krebsrisikos Zur Beantwortung dieser Fragen analysierte das Autorenteam die Daten von rund 46.000 Frauen, die bis zu 44 Jahre lang nachbeobachtet worden waren. Frauen, die jemals ein orales Kontrazeptivum einnahmen, hatten demnach ein geringeres Risiko für Kolorektal-, Endometrium- und Ovarialkarzinom sowie für lymphatische und hämatopoietische Krebserkrankungen (Inzidenzratenverhältnis [IRR| jeweils $0,81,0,66$, 0,67 beziehungsweise 0,74 ). Ein erhöhtes Risiko für Lungenkrebs wurde zwar ebenfalls beobachtet, allerdings galt dies nur für Anwenderinnen, die bereits zu Studieneintritt geraucht hatten.
Bei aktuellen und früheren Anwenderinnen wurde ein erhöhtes Risiko für Brustkrebs und Zervixkarzinom festgestellt (IRR jeweils 1,04 beziehungsweise 1,31$)$, doch dieses erhöhte Risiko schien fünf Jahre nach Beendigung der Einnahme wieder zu verschwinden. Außerdem zeigte sich keine Evidenz dafür, dass das Risiko für diese beiden Krebsformen zu einem späteren Zeitpunkt wieder anstieg, betonen die Autoren. Auch für das neue Auftreten eines erhöhten Krebsrisikos im höheren Alter wurde keine Evidenz gefunden. Das Fazit dieser Studie lautete daher: Frauen, die über viele Jahre hinweg orale Kontrazeptiva einnehmen, setzen sich keinem erhöhten Krebsrisiko aus. Bei manchen Krebsformen profitieren Anwenderinnen sogar von Risikosenkungen, die bis zu 30 Jahre nach Stopp der Einnahme noch zu beobachten sind.

\section{Fokus Brustkrebsrisiko}

Dem Thema Brustkrebsrisiko unter Einnahme oraler Kontrazeptiva widmete sich auch eine Forschergruppe der Universität Kopenhagen, deren Studie an knapp 1,8 Millionen Frauen im Alter zwischen 15 und 49 Jahren kürzlich im NEJM veröffentlicht wurde [2]. Auch hier zeigte sich ein erhöhtes relatives Brustkrebsrisiko von Anwenderinnen vs. Frauen, die niemals hormonale Kontrazeptiva eingenommen hatten (relatives Risiko 1,20). Das Risiko stieg mit der Dauer der Anwendung: von 1,09 bei einer Anwendung von kürzer als einem Jahr auf 1,38 bei einer Anwendung von mehr als zehn Jahren. Und: Auch nach Absetzung der hormonalen Kontrazeptiva war das Risiko bei jenen Frauen, die hormonale Kontrazeptiva fünf Jahre lang oder länger angewendet hatten, immer noch höher als bei Frauen, die keine hormonalen Kontrazeptiva angewendet hatten. Dieses Ergebnis sollte allerdings als, ,vorläufig " betrachtet werden, kommentiert der Autor des Leitartikels in derselben Ausgabe des NEJM [3]: Denn dieser Risikoanstieg wäre „nach Anpassung an Mehrfachvergleiche mit verschiedenen Kategorien von Dauer und Zeit seit der jeweils letzten Anwendung nicht signifikant gewesen".

Die absoluten Zahlen waren niedrig: Bei Anwendung eines beliebigen hormonellen Verhütungsmittels traten 13 zusätzliche Brustkrebsfälle pro 100.000 Personenjahre auf.Zudem müsse, fordert der Autor des Leitartikels, für jüngere Frauen das Brustkrebsrisiko gegenüber den anderen Vorteilen orale Kontrazeptiva abgewogen werden. Bei Frauen ab vierzig Jahren sollten hingegen aufgrund des erhöhten Brustkrebsrisikos (und der ebenfalls erhöhten, aber weniger häufigen Risiken wie Herzinfarkt oder Schlaganfall) alternative Verhütungsmethoden in Erwägung gezogen werden, wie etwa nicht-hormonale, reversible Kontrazeptiva mit Langzeitwirkung.

\section{Literatur}

1. Iversen L, Sivasubramaniam S, Lee AJ, Fielding S, Hannaford PC (2017) Lifetime cancer risk and combined oral contraceptives: the Royal College of General Practitioners' Oral Contraception Study. Am J Obstet Gynecol 216(6):580. e1-580.e9

2. Mørch LS, Skovlund CW, Hannaford PC, Iversen L, Fielding S, Lidegaard Ø (2017) Contemporary Hormonal Contraception and the Risk of Breast Cancer. N Engl J Med 377(23):2228-2239

3. Hunter DJ (2017) Oral Contraceptives and the Small Increased Risk of Breast Cancer. N Engl J Med 377(23):2276-2277

J. Gynäkol. Endokrinol. 2018 · 28:38 https://doi.org/10.1007/s41974-0180035-y

(c) Springer-Verlag GmbH Austria, ein Teil von Springer Nature 2018 